\title{
Géomatique et cartographie numérique
}

ou de la disponibilité des systèmes d'information à référence spatiale dans les bilbliothèques

\section{Geomatics and Numeric Cartography}

or the Availability of Spatial Information Systems

\section{Geomática y cartografía numérica}

\section{o la disponibilidad de los sistemas de información con referencia espacial (SIRS)}

\section{Pierre Lépine}

Volume 42, numéro 2, avril-juin 1996

URI : https://id.erudit.org/iderudit/1033285ar

DOI : https://doi.org/10.7202/1033285ar

Aller au sommaire du numéro

\section{Éditeur(s)}

Association pour l'avancement des sciences et des techniques de la documentation (ASTED)

ISSN

0315-2340 (imprimé)

2291-8949 (numérique)

Découvrir la revue

Citer cet article

Lépine, P. (1996). Géomatique et cartographie numérique : ou de la disponibilité des systèmes d'information à référence spatiale dans les bibliothèques. Documentation et bibliothèques, 42(2), 87-92. https://doi.org/10.7202/1033285ar

\section{Résumé de l'article}

Les systèmes d'information à référence spatiale " SIRS ", qui appartiennent à l'univers de la géomatique, ont donné naissance à la cartographie numérique. Après avoir décrit la création, les caractéristiques et le développement des SIRS, l'auteur établit des distinctions entre les types de données qui les composent et rappelle ensuite l'évolution de la cartographie manuelle à la cartographie numérique. Il poursuit l'examen de la structure des SIRS qui intègre la cartographie vectorielle et la cartographie matricielle. Enfin, il expose les changements provoqués par les SIRS dans les bibliothèques et les cartothèques ainsi que les rôles nouveaux que les cartothécaires seront appelés à jouer dans l'avenir.
Tous droits réservés ( $@$ Association pour l'avancement des sciences et des techniques de la documentation (ASTED), 1996
Ce document est protégé par la loi sur le droit d'auteur. L’utilisation des services d’Érudit (y compris la reproduction) est assujettie à sa politique d'utilisation que vous pouvez consulter en ligne.

https://apropos.erudit.org/fr/usagers/politique-dutilisation/ 


\title{
Géomatique et cartographie numérique ou de la disponibilité des systèmes d'information à référence spatiale dans les bibliothèques
}

\author{
Pierre Lépine \\ Section des cartes \\ Bibliothèque nationale du Québec
}

Les systèmes d'information à référence spatiale "SIRS», qui appartiennent à l'univers de la géomatique, ont donné naissance à la cartographie numérique. Après avoir décrit la création, les caractéristiques et le développement des SIRS, l'auteur établit des distinctions entre les types de données qui les composent et rappelle ensuite l'évolution de la cartographie manuelle à la cartographie numérique. II poursuit l'examen de la structure des SIRS qui intègre la cartographie vectorielle et la cartographie matricielle. Enfin, il expose les changements provoqués par les SIRS dans les bibliothèques et les cartothèques ainsi que les rôles nouveaux que les cartothécaires seront appelés à jouer dans l'avenir.

\section{Geomatics and Numeric Cartography or the Availability of Spatial Information Systems}

Spatial information systems, which belong to the domain of geomatics, gave rise to numeric cartography. Following a description of the creation, the characteristics, and the development of spatial information systems, the author outlines the types of information that make up these systems and describes the evolution of manual cartography towards numeric cartography. He describes the structure of these systems, which incorporate vectorial and matricial cartography. Finally, he outlines the changes these systems have had in libraries and map libraries and the new roles map librarians will be called to play.
Geomática y cartografia numérica o la disponibilidad de los sistemas de información con referencia espacial (SIRS)

Los sistemas de información con referencia espacial (SIRS), que pertenecen al universo de la geomática, han dado origen a la cartografía numérica. Después de haber descrito la creación, las características y el desarrollo de los SIRS, el autor establece las distinciones entre los tipos de datos que los componen y recuerda a continuación la evolución de la cartografía manual a la cartagrafía numérica. Continúa después su examen de la estructura de los SIRS, la cual se integra à la cartografía vectorial y al a cartografía de las matrices. Por fin, espone los cambios provocados por los SIRS en las bibliotecas y las cartotecas asi como las nuevas funciones que los cartotecarios tendran que efectuar en el futuro.
Un usager pourra bientôt se rendre à la bibliothèque de son choix et demander à consulter de nombreuses données dorénavant accessibles au public sous forme numérique. Installé à son ordinateur ou à celui de la bibliothèque, cet usager pourra alors manipuler ces données à sa guise aux fins de ses recherches; dans les cas où cette manipulation se fera à l'aide de systèmes d'information à référence spatiale (SIRS), l'usager pourra même, grâce à la cartographie numérique, qui est une des caractéristiques de ces systèmes, produire une carte géographique visualisant le résultat de ses recherches: une carte adaptée à ses besoins spécifiques (bien centrée, limitée à la région de son choix) et dans le format qui lui convient le mieux.
L'avènement des SIRS dans les bibliothèques permettra d'exploiter, outre les données géographiques et cartographiques traditionnelles, de grandes quantités d'informations que l'on n'associe pas généralement à la recherche géographique mais auxquelles on peut attribuer une valeur spatiale; on considère même que près de $65 \%$ de toutes les données actuellement compilées sous forme numérique à toutes sortes de fin peuvent être jumelées à des données localisables dans l'espace. De nombreuses données peuvent donc être étudiées au moyen des SIRS: c'est ce que font déjà beaucoup d'institutions commerciales, souvent à notre insu, avec des données publiques, comme celles du Recensement, et avec d'autres données privées qui nous concernent plus personnellement. On considère de plus en plus qu'il est souhaitable que les données tirées d'un milieu soient accessibles au plus grand nombre de personnes vivant dans ce milieu; et ce serait le rôle des bibliothèques de faciliter l'accès à ces données et à la technologie qui permet de les interpréter. Cette philosophie anime un grand nombre de nos collègues américains qui souhaitent l'implantation des SIRS dans toutes les grandes bibliothèques publiques; ils ont même déjà commencé à s'y préparer. Les SIRS, dont la cartographie numérique est une des manifestations les plus spectaculaires, appartiennent à un univers nouveau, celui de la géomatique. 
La géomatique et les systèmes d'information à référence spatiale

\section{La géomatique}

La géomatique se définit comme une discipline qui a pour objet la gestion de données à référence spatiale, c'està-dire la gestion de données auxquelles on peut attribuer une valeur de localisation géographique. Le développement de la géomatique a été rendu possible grâce à l'intégration des sciences et des technologies reliées à l'acquisition de ce type de données, à leur stockage, à leur traitement et à leur diffusion. La géographie et l'informatique figurent au premier plan de ces disciplines: le mot géomatique a d'ailleurs été formé par la contraction du radical géo- (comme géographie, géodésie...) et du mot informatique.

Les systèmes d'information à référence spatiale (SIRS)

La géomatique est née avec le développement de systèmes informatisés que l'on appelle des systèmes d'information à référence spatiale(SIRS). On les définit comme des systèmes d'information portant sur des données à référence spatiale. Les SIRS ont d'abord été désignés sous l'appellation de systèmes d'information géographique (SIG), ou, en anglais, Geographic Information System (GIS). Dans la documentation technique, on retrouve encore fréquemment ces appellation de SIG ou de GIS à la fois pour désigner les SIRS, ou même la géomatique dans son ensemble.

\section{La naissance des SIRS}

C'est à Hull, au Québec, dans les bureaux gouvernementaux fédéraux de l'Inventaire des terres du Canada (ITC), qu'a été conçu en 1970, un premier SIRS. L'ITC avait en effet à compiler une grande quantité d'informations géographiques sur le potentiel des terres canadiennes au niveau agricole, forestier, récréatif, etc. et il devait ensuite en faire la cartographie. Devant l'ampleur de la tâche, il a été décidé de travailler à développer un système informatisé pour répondre à ces besoins et c'est ce que l'on a fait. Ce premier SIRS s'est révélé à ce point intéressant qu'il a donné le coup d'envoi au développement de plusieurs autres systèmes similaires un peu partout dans le monde.

\section{Propriété des SIRS}

Un SIRS a la capacité d'emmagasiner et de traiter une très grande quantité de données: des données d'ordre géographique certes, mais aussi de nombreuses autres données qui peuvent toucher des domaines tout aussi variés que les domaines économique, social, juridique, etc. Un SIRS est aussi conçu de façon à pouvoir mettre toutes ces données en relation les unes avec les autres, grâce à ses fonctions d'analyse: analyse spatiale, analyse statistique, analyse d'images cartographiques... De façon plus importante encore, un SIRS a la propriété de permettre une forme d'interactivité avec ses utilisateurs: ces derniers peuvent faire varier les données en introduisant différents modèles ou scénarios et le système pourra communiquer les résultats sous forme de cartes, de graphiques et même sous forme de rapports.

\section{Expansion des SIRS}

II est rapidement apparu que l'information générée par les SIRS pouvait faciliter la prise de décision: dans le domaine de l'aménagement spatial, bien sûr, mais aussi dans de nombreux autres domaines qui, à prime abord, n'étaient pas visés par le développement des SIRS. Les SIRS se sont donc répandus rapidement, non seulement dans les administrations publiques d'où ils sont issus, mais aussi dans des milieux financiers et commerciaux.

À l'heure actuelle, on répertorie près de 190 logiciels différents de SIRS en comptant uniquement les logiciels commerciaux qui se veulent de portée universelle. La plupart des gouvernements se tournent maintenant vers les SIRS et, en même temps, se convertissent à la cartographie numérique. Au Québec, par exemple, il existe un Comité directeur de la géomatique qui voit à coordonner l'ensemble des projets gouvernementaux dans le domaine de la géomatique et de la cartographie numérique. Ce Comité a préparé, en 1992, un Plan géomatique gouvememental afin de mieux coordonner les efforts gouvernementaux dans ce domaine.
Les données à référence spatiale (DRS)

Si la géomatique se définit comme une discipline qui a pour objet la gestion de données à référence spatiale, et que les SIRS se définissent comme des systèmes d'information portant sur des données à référence spatiale, on peut se demander ce que sont ces dernières. Les données à référence spatiale (DRS) ou données géographiques sont des données auxquelles sont attribuées des valeurs de localisation spatiale ou de localisation géographique; elles ont parfois été désignées, au début, sous le nom de données géoréférencées. Les DRS comprennent des données descriptives, des données géométriques et des métadonnées.

\section{Les données descriptives}

Les données descriptives désignent d'abord les entités géographiques ellesmêmes, qu'elles soient topographiques, comme les continents, les lacs ou les îles ou encore qu'elles soient administratives, comme des municipalités ou des divisions électorales. Les données descriptives peuvent aussi être de nature qualitative ou de nature quantitative concernant des entités: des données sur la langue parlée par les habitants d'un territoire, par exemple, ou des données sur leur niveau de richesse.

\section{Les données géométriques}

Les données géométriques sont des données de nature mathématique qui renseignent sur la position et la forme des entités; elles servent à les situer dans l'espace. Les informations géométriques permettent de convertir en DRS de nombreuses données qui n'avaient pas nécessairement été compilées à l'origine comme telles. Les données géométriques comprennent des données telles que l'altitude, les coordonnées géographiques, etc. Nous reviendrons plus loin sur les données géométriques.

\section{Les métadonnées}

Les métadonnées renseignent enfin sur plusieurs autres aspects reliés aux données ou à leur traitement; il y a des métadonnées générales, comme celles 
concernant la date des données de base; il y a des métadonnées plus spécifiques qui concernent les normes de la présentation cartographique (la grosseur du trait ou la forme du symbole associé à telle ou telle donnée). Les métadonnées comprennent aussi les données bibliographiques: le titre du fichier de données ou celui des cartes que l'on peut en produire, le responsable de leur confection, la source des données; le mode de représentation des informations (de type vectoriel ou matriciel) ou encore des spécifications sur les outils (logiciels, équipements spéciaux) requis pour leur exploitation. Ce que l'on appelait autrefois en cartographie traditionnelle la légende de la carte. fait partie aujourd'hui des métadonnées.

\section{Cartographie manuelle et cartographie numérique}

La carte géographique, une représentation de données à référence spatiale

Une carte géographique, qu'elle soit manuelle ou numérique, est une représentation, sous forme de dessin, de données à référence spatiale. La représentation de ces données se fait à l'aide de signes et de symboles graphiques, en commençant par les points, les lignes, etc., mais en respectant un certain nombre de règles.

Avant de positionner une entité sur une carte, il aura d'abord fallu trouver l'emplacement exact qu'elle occupe dans l'espace; c'est au moyen de calculs, effectués sur un ou plusieurs points de cette entité, que l'on détermine sa localisation. $\mathrm{Ce}$ sont les cartographes, ou plus souvent les géomètres ou, au Québec, les arpenteurs-géomètres qui font de tels calculs à partir de levés effectués sur le terrain. Ils effectuent alors des calculs sur un ou des points déterminés de l'entité, qui deviennent ainsi des points de position: ce sont ces points qui, les premiers, seront reportés sur la carte.

L'adresse d'un point de position doit évidemment être non équivoque, ce qui suppose l'existence de repères fixes à partir desquels on puisse effectuer son calcul. Les tout premiers repères sont d'autres points que l'on nomme des points d'origine. Actuellement, sur la Terre, les points d'origine conventionnels sont, pour chacune des trois dimensions, les suivants: un point quelconque situé sur un parallèle encerclant l'équateur terrestre pour le calcul de la latitude; un point quelconque situé sur un méridien passant par l'Observatoire de Greenwich, en banlieue de Londres, pour le calcul de la longitude; et enfin un point calculé en fonction du niveau moyen de la surface des mers, le géoïde, pour le calcul de l'altitude. La latitude et la longitude (mais non pas l'altitude) déterminent ce que l'on appelle les coordonnées géographiques.

La détermination des coordonnées géographiques au temps de la Nouvelle-France...

Les méthodes pour déterminer les coordonnées géographiques ont beaucoup évolué dans le temps. Par exemple, à l'époque de Samuel de Champlain (ca 1567-1635), qui est l'explorateur et le cartographe le plus illustre de la NouvelleFrance, faire un levé était autrement plus compliqué qu'aujourd'hui. Avec un astrolabe, Champlain prenait une première mesure de l'angle du soleil; prenant en considération l'heure et la date de son observation, il pouvait ensuite, par une série de calculs, déterminer la latitude du lieu où il se trouvait.

Champlain devait ensuite déterminer la longitude de ce lieu, en situant son emplacement par rapport à l'île de Fer, une île des Canaries située à $16^{\circ}$ à l'ouest de Greenwich: c'est cette île, et non pas l'observatoire Iondonien de Greenwich, qui servait alors le plus fréquemment de point d'origine pour le calcul de la longitude. Pour calculer avec précision la longitude par rapport à cet endroit, il eût fallu connaître simultanément l'heure à l'île de Fer et celle du lieu de son observation; mais à l'époque de Champlain, il n'existait pas d'horloge qui pût conserver pendant plusieurs semaines ou plusieurs mois l'heure d'un point situé à une aussi grande distance. En réalité, les calculs de longitude effectués par Champlain vont refléter une erreur moyenne de $3^{\circ}$ par rapport à l'île de Fer.

Malgré tout, Champlain aura réussi à réaliser les premières cartes de la Nouvelle-France oùl'on reconnaît, entre autres, la forme géométrique qui caractérise cette partie du territoire qui est devenu le Québec d'aujourd'hui.
... et la localisation d'un point géographique à l'ère des satellites

La facilité avec laquelle on peut maintenant obtenir la position de quelque point géographique que ce soit sur la Terre aurait eu de quoi déconcerter Champlain. En effet, grâce au système de positionnement global (SPG) ou système de localisation GPS (de l'anglais Global Positioning System), qui repose sur des données géospatiales satellitaires, on peut maintenant connaître, en quelques minutes et en tout lieu, la position précise de l'endroit où l'on effectue un levé: latitude, longitude et même altitude et le tout à quelques centimètres près. Pour avoir accès à ces données, il suffit de disposer d'un récepteur qui permet de capter des signaux radio émis par une série de satellites situés dans l'espace; certains de ces appareils, bien qu'un peu moins précis, sont des appareils portatifs, sans doute ne sont-ils pas plus encombrants à transporter que les instruments dont Samuel de Champlain disposait à l'époque de ses nombreuses pérégrinations nordaméricaines.

Les projections, les mesures de la Terre et les systèmes de référence NAD 27 et NAD 83

Comment s'y prend-on pour représenter une sphère (la Terre) qui est en trois dimensions, sur une surface plane (une carte) qui n'a que deux dimensions et s'assurer en même temps d'une correspondance entre les points du plan et les points réels à la surface de la sphère. C'est toute la question des projections. Cette question très technique est relativement complexe et concerne aussi bien la cartographie manuelle traditionnelle que la cartographie numérique, cependant nous ne nous y attarderons pas dans le cadre de cet article.

Un autre aspect technique important pour la cartographie et qui peut influencer le positionnement des entités est celui de la détermination de la forme et des dimensions de la Terre: c'est une question qui relève, en fait, de la géodésie. Sans s'attarder à cet aspect, mentionnons que la mise en orbite dans les trente dernières années de satellites permettant des mesures très précises de la Terre a entraîné la vérification et la 
rectification de nombreux calculs antérieurs. On a dû, entre autres, remplacer l'ancien système de référence géodésique connu sous le nom de North American Datum 1927 (NAD 27), par un autre que l'on désigne en français comme le Système de référence nord-américain de 1983 (NAD 83). L'adoption de ce système permettant d'exprimer de façon univoque la position de tout point en Amérique du Nord a obligé à modifier les coordonnées de plusieurs points géodésiques, des points qui servent de repères pour le calcul de toutes les autres entités.

La carte et la représentation des données sous forme géométrique

Revenons donc à la carte dont on a dit qu'elle était composée de signes et de symboles graphiques. La carte traditionnelle ou manuelle est une image composée de points reliés entre eux formant des lignes ou vecteurs et de lignes reliées entre elles pouvant former des polygones. La présentation de ces données, en particulier sous forme de polygones représentant des surfaces ou des aires géographiques, fait donc appel à la géométrie. C'est bien grâce à la géométrie et grâce aux mathématiques en général que l'on pourra, au moyen des SIRS, effectuer de nombreuses analyses sur les données géographiques.

Topologie, cartographie numérique et structures des SIRS

L'intérêt majeur des SIRS réside d'abord dans leur capacité à calculer des données mathématiques concernant des phénomènes géographiques, à les comparer et à générer de nouvelles données géographiques. C'est que les SIRS permettent l'application, en géomatique, des principes de la topologie, une branche des mathématiques traitant des relations de voisinage qui s'établissent entre des figures géométriques. L'utilisation de la topologie facilite non seulement l'analyse des DRS, mais elle en facilite aussi leur représentation cartographique: c'est donc dire que l'on peut se servir des SIRS pour produire des cartes de façon automatisée, c'est-à-dire pour faire de la cartographie numérique.
Selon les besoins d'analyse et de cartographie, les SIRS peuvent enregistrer et traiter les données selon deux modes différents: soit en se servant d'une structure vectorielle (vector structure, en anglais), soit en se servant d'une structure matricielle (raster structure). Selon la structure interne utilisée, les cartes numériques produites au moyen des SIRS le seront sous forme d'images vectorielles ou sous forme d'images matricielles.

\section{Les SIRS et la cartographie vectorielle}

Une image vectorielle est une image dont tous les vecteurs sont obtenus en reliant entre eux des points: l'emplacement de ces points aura été déterminé selon leurs coordonnées (dans l'axe des $x$ et des $y$ ). En reliant ces points, on donne naissance à des lignes ou vecteurs et à des polygones. Dans un SIRS fonctionnant selon une structure vectorielle, l'ordinateur pourra concevoir toute une série de polygones et en faire leur analyse. Pour la représentation cartographique, l'ordinateur partira des points de numérisation, les reliera entre eux et formera tous les polygones nécessaires à la représentation des surfaces cartographiées.

Dans une structure vectorielle, et avec des images vectorielles, on suppose que chaque entité géographique, chaque aire géographique peut être représentée par un polygone délimité de façon très nette par une ligne qui le sépare d'une autre entité contiguë; c'est vrai pour marquer une île ou un lac et c'est encore plus vrai pour montrer des limites administratives comme des frontières ou des lignes de propriété (le cadastre). Les images vectorielles peuvent être très précises et très utiles pour montrer des phénomènes linéaires comme des routes ou des cours d'eau (ruisseaux, rivières). Elles sont beaucoup moins appropriées cependant pour des phénomènes dont les limites ne sont pas à strictement parler tranchées au couteau: la base d'une montagne, l'étendue d'une essence forestière en forêt, la limite entre deux catégories de sols. II s'agit là de phénomènes pour lesquels un autre type de structure, la structure matricielle, sera davantage appropriée, tant pour des fonctions d'analy- se que pour des modalités de représentation cartographique.

\section{Les SIRS et la cartographie matricielle}

Les images matricielles sont des images constituées de cellules; ces cellules, toutes contiguës, sont rectangulaires (elles peuvent être carrées). Au lieu donc que ce soit le point qui soit l'unité d'origine de toute la représentation, c'est la cellule. Alors que dans une structure vectorielle l'emplacement du point est déterminé selon ses coordonnées (dans l'axe des $x$ et des $y$ ), dans une structure matricielle, la position de la cellule est déterminée selon son emplacement sur une grille régulière ou matrice dans un ordre différent: soit de gauche à droite et de haut en bas. Les cellules sont aussi parfois appelées pixels (de l'anglais, picture element).

Dans une structure matricielle et avec des images matricielles, l'ordinateur reçoit une information pour chaque cellule, même si cette information est exactement la même pour des centaines ou des milliers de cellules contiguës: il apparaît donc, dans un premier temps, que ce mode a pour effet d'accroître inutilement le nombre de données: il y a apparence de «pollution» de l'information. Par contre, une fois leur dimension déterminée, le nombre des cellules d'une structure matricielle demeure constant, contrairement à la structure vectorielle où chaque ajout de données peut provoquer de nouveaux recoupements de polygones et donc la multiplication de leurs intersections enregistrées sous forme de points.

Les appareils qui font de la numérisation par balayage, (ou scanning, en anglais), décomposent ce qu'ils captent en une série de cellules: ils enregistrent donc les données dans un mode matriciel, ce qui permet à des SIRS fonctionnant selon ce mode de les traiter. Les informations traitées en mode matriciel par l'ordinateur pourront ensuite servir à la production de cartes numériques qui seront matricielles.

La superposition des images vectorielles et des images matricielles

Depuis peu, les SIRS permettent la superposition des images vectorielles et 
des images matricielles: certaines cellules de l'image matricielle peuvent ainsi être jumelées à des points de l'image vectorielle et vice-versa. L'objectif est de pouvoir transférer certains attributs des données acquises selon un type de structure vers des données acquises selon un autre type. Il existe effectivement une quantité phénoménale d'informations qui n'arrivent actuellement que sous forme d'images matricielles, les images satellitaires par exemple; la conversion de ces DRS d'une forme à une autre est devenue nécessaire pour qu'elles puissent vraiment être exploitées de façon optimale.

\section{Le dessin assisté par ordinateur}

Il faut mentionner que les cartographes ont aussi une autre façon de produire des cartes numériques sans nécessairement passer par les SIRS; ils utilisent des logiciels de dessin, ou de dessin assisté par ordinateur (computer-aided design ou $C A D$, en anglais): ces logiciels sont orientés vers la réalisation de cartes d'une grande qualité graphique, mais ne constituent pas en soi des SIRS. II est maintenant possible d'établir des ponts entre les SIRS et les logiciels de dessin assisté par ordinateur afin de profiter des avantages des uns et des autres.

\section{La cartographie thématique}

La combinaison d'une grande quantité de données au moyen des SIRS permet un nouvel essor de la cartographie thématique qui consiste en la réalisation de cartes dans lesquelles la représentation des phénomènes est faite en fonction de variables d'ordre qualitatif ou quantitatif. Le développement de la cartographie thématique permet de répondre à des besoins plus pointus ou à des attentes plus personnelles aussi bien des profanes que des chercheurs.

Les SIRS et la cartographie dans les bibliothèques et les cartothèques

Actuellement, les grandes bibliothèques font l'acquisition des cartes géographiques et les regroupent dans leurs cartothèques. Les usagers de ces cartothèques peuvent consulter entre autres les grandes séries de cartes officielles préparées et imprimées par les principaux producteurs privés et gouvernementaux.
Plusieurs cartes maintenant conçues au moyen des SIRS, notamment les séries les plus générales, même disponibles sous forme numérique, continuent d'être distribuées sous forme imprimée. D'autres, cependant, ne sont disponibles que sous une forme numérique.

Les cartothécaires sont conscients que de plus en plus de leurs utilisateurs, formés ou non aux ressources de la géomatique, demanderont à consulter les données à référence spatiale directement dans leur forme numérique; car ce sont les utilisateurs eux-mêmes qui, après manipulation de ces DRS avec leur ordinateur ou celui de la cartothèque, créeront leurs propres cartes, des cartes qui répondront davantage à leurs besoins spécifiques. Bien sûr, tous les logiciels de SIRS ne sont pas conviviaux et ne se retrouveront pas dans les bibliothèques: mais déjà, il existe des visualisateurs qui, sans permettre d'analyses sophistiquées des DRS, en permettent néammoins leur visualisation sous forme de cartes.

La production et la commercialisation de fichiers et de bases de données à référence spatiale

Les producteurs traditionnels de cartes, les agences gouvernementales entre autres, sont donc devenus des producteurs et des diffuseurs de DRS. Ces données sont disponibles soit sous forme de fichiers correspondant à la quantité de données nécessaires pour permettre à l'ordinateur de produire une seule carte à l'écran, soit sous forme de bases de données à référence spatiale (BDRS) comprenant un ensemble de données pour un territoire défini et contenant l'équivalent d'un grand nombre de cartes traditionnelles.

Dès maintenant, au Québec, on peut obtenir sous forme de données numériques, l'équivalent d'un feuillet traditionnel sur fichier informatique à un coût d'environ trois cents dollars; un fichier peut être contenu sur une ou deux disquettes à haute densité. À ce prix cependant, pour obtenir les données représentant la totalité des feuillets de la carte de base à l'échelle de 1:20 000, soit près de 2000 feuillets, il faudrait débourser une somme d'argent assez considérable. Alors qu'une carte sur papier coûte souvent moins de dix dollars, les DRS qui composent les cartes numériques sont donc vendues à un prix très élevé mais leur utilisation, bien sûr, n'est pas la même. Le coût des fichiers de DRS est cependant à la baisse et pourrait continuer à baisser. Leur accessibilité sur disques optiques compacts (DOC), aussi appelés CD-ROM, pourrait les rendre encore plus attrayantes. Avec l'accroissement presqu'effréné de données de toutes sortes, avec leur accessibilité accrue, notamment par l'autoroute électronique, plusieurs estiment que leur prix ne peut que chuter considérablement. Cette surenchère pourrait même aboutir à une accessibilité sans frais d'un très grand nombre de données.

La diffusion et la commercialisation des DRS soulèvent certains problèmes qui ne sont pas seulement rattachés à leur acquisition et à leur coût, mais aussi à leur utilisation: compatibilité des données avec tel ou tel type de logiciel de SIRS, respect des droits d'auteur, fiabilité et intégrité des données après manipulation par les utilisateurs, sans compter la rupture de la confidentialité par le rapprochement de données qui, séparément, paraissent assez inoffensives pour la vie privée des gens.

Un nouveau rôle pour les cartothèques et les bibliothèques

Comme il est prévu que les clients traditionnels des cartothèques deviendront des consommateurs de données à référence spatiale, on ne pourra pas seulement se contenter de leur offrir un choix pertinent de DRS; encore faudra-t-il mettre à leur disposition l'équipement nécessaire (ordinateurs, logiciels de SIRS) et leur assurer un soutien personnalisé. Pour que l'usager soit lui-même en mesure de préparer à l'écran la carte affichant le résultat de l'analyse des données mises à sa disposition, il devra pouvoir compter sur des ressources pour l'aider dans ses démarches. Aux États-Unis, où l'on veut promouvoir la diffusion et l'utilisation des DRS, on a déjà mis sur pied, à l'intention des cartothécaires; des programmes d'alphabétisation aux SIRS (GIS Literacy Program); ce programme vient même d'être étendu aux cartothécaires canadiens. Si le personnel des cartothèques 
est le premier visé, on souhaite que ce programme dépasse le milieu universitaire où l'on retrouve d'abord les cartothèques pour s'étendre éventuellement à toutes les grandes bibliothèques publiques. Étant donné, en effet, l'impact majeur des SIRS, on souhaite que tous les citoyens puissent avoir accès à la fois aux données et aux SIRS.

\section{En conclusion}

L'avènement des SIRS et de la cartographie numérique et plus spécialement de la diffusion de nombreuses données à référence spatiales (DRS) constituées en fichiers ou en bases de données à référence spatiale (BDRS) pourrait transformer le rôle traditionnel des bibliothèques: pour rendre accessible DRS et SIRS à tous les citoyens, les grandes bibliothèques seront donc bientôt appelées à tenir compte d'une réalité nouvelle, celle de la géomatique.

\section{Sources}

Association cartographique internationale. Dictionnaire multilingue de termes techniques cartographiques. Wiesbaden: Franz Steiner Verlag, 1973. $573 \mathrm{p}$.

Bergeron, Marcel. Vocabulaire de la géomatique. Québec: Publications du Québec, 1993.46 p.

Berry, Joseph K. Beyond mapping: concepts, algorithms and issues in GIS. Fort Collins, Colorado: GIS World, 1993.246 p.+1 logiciel d'apprentissage.

Environmental Systems Research Institute. GIS in libraries: public access to GIS. ESRI, 1994. 1 vidéo cassette.

Joly, Fernand. La cartographie. Que sais-je?, ${ }^{\circ}$ 937. Paris: PUF, 1985. 128 p.

Québec. Ministère du Conseil exécutif. Comité directeur du plan géomatique gouvernemental. Géo info. Québec: Le Ministère, 1989.

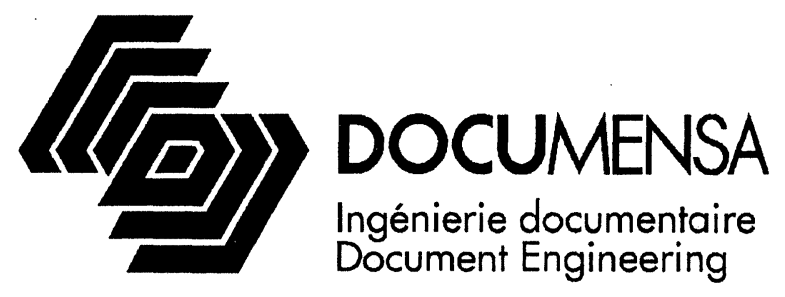

\section{Votre partenaire SGML-HTML au Québec}

Votre fond documentaire est la mémoire et le savoir de votre organisation. Sa structuration, sa gestion et sa diffusion sont des enjeux majeurs de votre productivité concurrentielle. DOCUMENSA met son expertise à votre service pour concevoir et implanter votre système documentaire conforme à la norme SGML. Vous disposez ainsi d'une source unique, réutilisable et indépendante des fournisseurs pour:

- La pérennité de vos informations stratégiques

- La mise en place des meilleurs outils logiciels de gestion documentaire

- Une chaîne éditoriale SGML intégrée pour la production de documents

- La diffusion multi-support de vos banques d'information sur:

- DOC (CD-ROM);

- I'autoroute de l'information en HTML et SGML sur le Web;

- publication imprimée;

- publication braille et vocale.

\section{DOCUMENSA}

l'ingénierie documentaire en action

Tél.: (514) 524-7722email: documen@cam.org

Tlc.: (514) 524-5441Web : http://www.cam.org/ documen 